\title{
EDGE IDEALS OF CLIQUE CLUTTERS OF COMPARA- BILITY GRAPHS AND THE NORMALITY OF MONOMIAL IDEALS
}

\author{
LUIS A. DUPONT and RAFAEL H. VILLARREAL*
}

\begin{abstract}
The normality of a monomial ideal is expressed in terms of lattice points of blocking polyhedra and the integer decomposition property. For edge ideals of clutters this property characterizes normality. Let $G$ be the comparability graph of a finite poset. If $\operatorname{cl}(G)$ is the clutter of maximal cliques of $G$, we prove that $\operatorname{cl}(G)$ satisfies the max-flow min-cut property and that its edge ideal is normally torsion free. Then we prove that edge ideals of complete admissible uniform clutters are normally torsion free.
\end{abstract}

\section{Introduction}

Let $R=K\left[x_{1}, \ldots, x_{n}\right]$ be a polynomial ring over a field $K$ and let $I$ be a monomial ideal of $R$. We are interested in determining what families of monomial ideals have the property that $I$ is normal or normally torsion free. An aim here is to explain how these two algebraic properties interact with combinatorial optimization and linear programming problems. Recall that $I$ is called normal (resp. normally torsion free) if $I^{i}=\overline{I^{i}}$ (resp. $I^{i}=I^{(i)}$ ) for all $i \geq 1$, where $\overline{I^{i}}$ and $I^{(i)}$ denote the integral closure of the $i$ th power of $I$ and the $i$ th symbolic power of $I$ respectively (see the beginning of Sections 2 and 4 for the precise definitions of $\overline{I^{i}}$ and $I^{(i)}$ ). If $I=\bar{I}$, the ideal $I$ is called integrally closed.

The contents of this paper are as follows. In Section 2 we study the normality of monomial ideals. We are able to characterize this property in terms of blocking polyhedra and the integer decomposition property (see Theorem 2.1). For integrally closed ideals this property characterizes normality (see Corollary 2.2). As a consequence, using a result of Baum and Trotter [2], we describe the normality of a monomial ideal in terms of the integer rounding property (see Corollary 2.5).

Before introducing the main results of Sections 3 and 4, let us recall some notions that will play an important role in what follows. Let $\mathscr{C}$ be a clutter with

\footnotetext{
${ }^{*}$ RFH partially supported by CONACyT grant $49251-\mathrm{F}$ and SNI.

Received 24 June 2008, in revised form 23 October 2008.
} 
finite vertex set $X=\left\{x_{1}, \ldots, x_{n}\right\}$, that is, $\mathscr{C}$ is a family of subsets of $X$, called edges, none of which is included in another. The set of vertices and edges of $\mathscr{C}$ are denoted by $V(\mathscr{C})$ and $E(\mathscr{C})$ respectively. The incidence matrix of $\mathscr{C}$ is the vertex-edge matrix whose columns are the characteristic vectors of the edges of $\mathscr{C}$. The edge ideal of $\mathscr{C}$, denoted by $I(\mathscr{C})$, is the ideal of $R$ generated by all monomials $\prod_{x_{i} \in e} x_{i}$ such that $e \in E(\mathscr{C})$.

Let $P=(X, \prec)$ be a partially ordered set (poset for short) on the finite vertex set $X$ and let $G$ be its comparability graph. Recall that the vertex set of $G$ is $X$ and the edge set of $G$ is the set of all unordered pairs of vertices $\left\{x_{i}, x_{j}\right\}$ such that $x_{i}$ and $x_{j}$ are comparable. A clique of $G$ is a subset of the set of vertices of $G$ that induces a complete subgraph. The clique clutter of $G$, denoted by $\operatorname{cl}(G)$, is the clutter with vertex set $X$ whose edges are exactly the maximal cliques of $G$ (maximal with respect to inclusion).

Our main algebraic result is presented in Section 4. It shows that the edge ideal $I=I(\operatorname{cl}(G))$ of $\operatorname{cl}(G)$ is normally torsion free (see Theorem 4.2). To prove this result we first show that the clique clutter of $G$ has the max-flow min-cut property (see Theorem 3.7). Then we use a remarkable result of [7] showing that an edge ideal $I(\mathscr{C})$, of a clutter $\mathscr{C}$, is normally torsion free if and only if $\mathscr{C}$ has the max-flow min-cut property. As an application, we prove that edge ideals of complete admissible uniform clutters are normally torsion free (see Theorem 4.3). This interesting family of clutters was introduced and studied in [5].

Along the paper we introduce most of the notions that are relevant for our purposes. Our main references for combinatorial optimization and commutative algebra are [3], [12], [14], [15]. In these references the reader will find the undefined terminology and notation that we use in what follows.

\section{Normality of monomial ideals}

Let $R=K\left[x_{1}, \ldots, x_{n}\right]$ be a polynomial ring over a field $K$, let $I$ be a monomial ideal of $R$ generated by $x^{v_{1}}, \ldots, x^{v_{q}}$, and let $A$ be the $n \times q$ matrix with column vectors $v_{1}, \ldots, v_{q}$. As usual, we will use $x^{a}$ as an abbreviation for $x_{1}^{a_{1}} \ldots x_{n}^{a_{n}}$, where $a=\left(a_{i}\right)$ is a vector in $\mathrm{N}^{n}$. Recall that the integral closure of $I^{i}$, denoted by $\overline{I^{i}}$, is the ideal of $R$ given by

$$
\overline{I^{i}}=\left(\left\{x^{a} \in R \mid \exists p \in \mathbf{N} \backslash\{0\} ;\left(x^{a}\right)^{p} \in I^{p i}\right\}\right),
$$

see for instance [15, Proposition 7.3.3]. The ideal $I$ is called normal if $I^{i}=\overline{I^{i}}$ for $i \geq 1$. In this section we give a characterization of the normality of $I$ in terms of lattice points of blocking polyhedra. The polyhedron

$$
Q=Q(A)=\{x \mid x \geq 0 ; x A \geq \mathbf{1}\}
$$


is called the covering polyhedron of $I$. As usual, we denote the vector $(1, \ldots, 1)$ by 1 . If $a=\left(a_{1}, \ldots, a_{n}\right)$ and $b=\left(b_{1}, \ldots, b_{n}\right)$ are vectors, we write $a \leq b$ if $a_{i} \leq b_{i}$ for all $i$. The polyhedron

$$
B(Q)=\{z \mid z \geq 0 ;\langle z, x\rangle \geq 1 \text { for all } x \text { in } Q\}
$$

is called the blocking polyhedron of $Q=Q(A)$. Here $\langle$,$\rangle denotes the standard$ inner product in $\mathrm{R}^{n}$. The polyhedron $B(Q)$ is said to have the integer decomposition property if for each natural number $k$ and for each integer vector $a$ in $k B(Q), a$ is the sum of $k$ integer vectors in $B(Q)$; see [12, pp. 66-82].

THEOREM 2.1. The ideal I is normal if and only if the blocking polyhedron $B(Q)$ of $Q=Q(A)$ has the integer decomposition property and all minimal integer vectors of $B(Q)$ are columns of $A$ (minimal with respect to $\leq$ ).

Proof. First we show the equality $B(Q)=\mathrm{R}_{+}^{n}+\operatorname{conv}\left(v_{1}, \ldots, v_{q}\right)$, where "conv" stands for convex hull and $\mathrm{R}_{+}=\{x \in \mathrm{R} \mid x \geq 0\}$. The right hand side is clearly contained in the left hand side. Conversely take $z$ in $B(Q)$, then $\langle z, x\rangle \geq 1$ for all $x \in Q(A)$ and $z \geq 0$. Let $\ell_{1}, \ldots, \ell_{r}$ be the vertex set of $Q(A)$. In particular $\left\langle z, \ell_{i}\right\rangle \geq 1$ for all $i$. Then $\left\langle(z, 1),\left(\ell_{i},-1\right)\right\rangle \geq 0$ for all $i$. From [7, Theorem 3.2] we get that $(z, 1)$ belongs to the cone generated by

$$
\mathscr{A}^{\prime}=\left\{e_{1}, \ldots, e_{n},\left(v_{1}, 1\right), \ldots,\left(v_{q}, 1\right)\right\} .
$$

Thus $z$ is in $\mathrm{R}_{+}^{n}+\operatorname{conv}\left(v_{1}, \ldots, v_{q}\right)$. This completes the proof of the asserted equality. Hence $B(Q) \cap \mathrm{Q}^{n}=\mathrm{Q}_{+}^{n}+\operatorname{conv}_{\mathrm{Q}}\left(v_{1}, \ldots, v_{q}\right)$ because the polyhedron $B(Q)$ is rational. Using this equality and the description of the integral closure given in Eq. (1), we readily obtain the equality

$$
\overline{I^{k}}=\left(\left\{x^{a} \mid a \in k B(Q) \cap Z^{n}\right\}\right)
$$

for $0 \neq k \in \mathrm{N}$. Assume that $I$ is normal, i.e., $\overline{I^{k}}=I^{k}$ for $k \geq 1$. Let $a$ be an integer vector in $k B(Q)$. Then $x^{a} \in I^{k}$ and consequently $a$ is the sum of $k$ integer vectors in $B(Q)$, that is, $B(Q)$ has the integer decomposition property. Take a minimal integer vector $a$ in $B(Q)$. Then $x^{a} \in \bar{I}=I$ and we can write $a=\delta+v_{i}$ for some $v_{i}$ and for some $\delta \in \mathrm{N}^{n}$. Thus $a=v_{i}$ by the minimality of $a$. Conversely assume that $B(Q)$ has the integer decomposition property and all minimal integer vectors of $B(Q)$ are columns of $A$. Take $x^{a} \in \overline{I^{k}}$, i.e., $a$ is an integer vector of $k B(Q)$. Hence $a$ is the sum of $k$ integer vectors $\alpha_{1}, \ldots, \alpha_{k}$ in $B(Q)$. Since any minimal vector of $B(Q)$ is a column of $A$ we may assume that $\alpha_{i}=c_{i}+v_{i}$ for $i=1, \ldots, k$. Hence $x^{a} \in I^{k}$, as required.

COROLlary 2.2. If $I=\bar{I}$, then $I$ is normal if and only if the blocking polyhedron $B(Q)$ has the integer decomposition property. 
Proof. $\Rightarrow$ ) If $I$ is normal, by Theorem 2.1 the blocking polyhedron $B(Q)$ has the integer decomposition property.

$\Leftarrow)$ Take $x^{a} \in \overline{I^{k}}$. From Eq. (2) we get that $a$ is an integer vector of $k B(Q)$. Hence $a$ is the sum of $k$ integer vectors $\alpha_{1}, \ldots, \alpha_{k}$ in $B(Q)$. Using Eq. (2) with $k=1$, we get that $\alpha_{1}, \ldots, \alpha_{k}$ are in $\bar{I}=I$. Hence $x^{a} \in I^{k}$, as required.

Corollary 2.3. If $I=I(\mathscr{C})$ is the edge ideal of a clutter $\mathscr{C}$, then $I$ is normal if and only if the blocking polyhedron $B(Q)$ has the integer decomposition property.

Proof. Recall that $I$ is an intersection of prime ideals (see [15, Corollary 5.1.5]). Thus it is seen that $\bar{I}=I$. Then the result follows from Corollary 2.2.

Definition 2.4. The system $x \geq 0 ; x A \geq \mathbf{1}$ of linear inequalities is said to have the integer rounding property if

$$
\max \left\{\langle y, \mathbf{1}\rangle \mid y \geq 0 ; A y \leq w ; y \in \mathbf{N}^{q}\right\}=\lfloor\max \{\langle y, \mathbf{1}\rangle \mid y \geq 0 ; A y \leq w\}\rfloor
$$

for each integer vector $w$ for which the right hand side is finite.

Systems with the integer rounding property have been widely studied; see for instance [11, Chapter 22, pp. 336-338], [12, pp. 82-83], and the references there.

COROLlary 2.5. I is a normal ideal if and only if the system $x A \geq \mathbf{1} ; x \geq 0$ has the integer rounding property.

Proof. According to [2] the system $x A \geq \mathbf{1} ; x \geq 0$ has the integer rounding property if and only if the blocking polyhedron $B(Q)$ of $Q=Q(A)$ has the integer decomposition property and all minimal integer vectors of $B(Q)$ are columns of $A$ (minimal with respect to $\leq$ ) (cf. [12, p. 82, Eq. (5.80)]). Thus the result follows at once from Theorem 2.1.

There are some other useful characterizations of the normality of a monomial ideal [4, Theorem 4.4].

\section{Maximal cliques of comparability graphs}

In this section we introduce the max-flow min-cut property and prove our main combinatorial result, that is, we prove that the clique clutter of a comparability graph satisfies the max-flow min-cut property.

Definition 3.1. Let $\mathscr{C}$ be a clutter and let $A$ be its incidence matrix. The clutter $\mathscr{C}$ satisfies the max-flow min-cut property if both sides of the LP-duality equation

$$
\min \{\langle w, x\rangle \mid x \geq 0 ; x A \geq \mathbf{1}\}=\max \{\langle y, \mathbf{1}\rangle \mid y \geq 0 ; A y \leq w\}
$$


have integer optimum solutions $x$ and $y$ for each non-negative integer vector $w$.

Let $\mathscr{C}$ be a clutter on the vertex set $X=\left\{x_{1}, \ldots, x_{n}\right\}$. A set of edges of $\mathscr{C}$ is called independent or stable if no two of them have a common vertex. A subset $C \subset X$ is called a minimal vertex cover of $\mathscr{C}$ if: (i) every edge of $\mathscr{C}$ contains at least one vertex of $C$, and (ii) there is no proper subset of $C$ with the first property. We denote the smallest number of vertices in any minimal vertex cover of $\mathscr{C}$ by $\alpha_{0}(\mathscr{C})$ and the maximum number of independent edges of $\mathscr{C}$ by $\beta_{1}(\mathscr{C})$. These two numbers satisfy $\beta_{1}(\mathscr{C}) \leq \alpha_{0}(\mathscr{C})$.

Definition 3.2. If $\beta_{1}(\mathscr{C})=\alpha_{0}(\mathscr{C})$, we say that $\mathscr{C}$ has the König property.

Let $x_{i}$ be a vertex of $\mathscr{C}$. Then duplicating $x_{i}$ means extending $X$ by a new vertex $x_{i}^{\prime}$ and replacing $E(\mathscr{C})$ by

$$
E(\mathscr{C}) \cup\left\{\left(e \backslash\left\{x_{i}\right\}\right) \cup\left\{x_{i}^{\prime}\right\} \mid x_{i} \in e \in E(\mathscr{C})\right\} .
$$

The deletion of $x_{i}$, denoted by $\mathscr{C} \backslash\left\{x_{i}\right\}$, is the clutter formed from $\mathscr{C}$ by deleting the vertex $x_{i}$ and all edges containing $x_{i}$. A clutter obtained from $\mathscr{C}$ by a sequence of deletions and duplications of vertices is called a parallelization. If $w=\left(w_{i}\right)$ is a vector in $\mathbf{N}^{n}$, we denote by $\mathscr{C}^{w}$ the clutter obtained from $\mathscr{C}$ by deleting any vertex $x_{i}$ with $w_{i}=0$ and duplicating $w_{i}-1$ times any vertex $x_{i}$ if $w_{i} \geq 1$.

The notion of parallelization can be used to give the following characterization of the max-flow min-cut property which is suitable to study the clique clutter of the comparability graph of a poset.

Theorem 3.3. [12, Chapter 79, Eq. (79.1)] Let $\mathscr{C}$ be a clutter. Then $\mathscr{C}$ satisfies the max-flow min-cut property if and only if $\beta_{1}\left(\mathscr{C}^{w}\right)=\alpha_{0}\left(\mathscr{C}^{w}\right)$ for all $w \in \mathbf{N}^{n}$.

Lemma 3.4. Let $\mathrm{cl}(G)$ be the clutter of maximal cliques of a graph $G$. If $G^{1}\left(\right.$ resp. $\left.\operatorname{cl}(G)^{1}\right)$ is the graph (resp. clutter) obtained from $G($ resp. $\operatorname{cl}(G))$ by duplicating the vertex $x_{1}$, then $\operatorname{cl}(G)^{1}=\operatorname{cl}\left(G^{1}\right)$.

Proof. Let $y_{1}$ be the duplication of $x_{1}$. Set $\mathscr{C}=\operatorname{cl}(G)$. First we prove that $E\left(\mathscr{C}^{1}\right) \subset E\left(\operatorname{cl}\left(G^{1}\right)\right)$. Take $e \in E\left(\mathscr{C}^{1}\right)$. Case (i): Assume $y_{1} \notin e$. Then $e \in E(\mathscr{C})$. Clearly $e$ is a clique of $G^{1}$. If $e \notin E\left(\operatorname{cl}\left(G^{1}\right)\right)$, then $e$ can be extended to a maximal clique of $G^{1}$. Hence $e \cup\left\{y_{1}\right\}$ must be a clique of $G^{1}$. Note that $x_{1} \notin e$ because $\left\{x_{1}, y_{1}\right\}$ is not an edge of $G^{1}$. Then $e \cup\left\{x_{1}\right\}$ is a clique of $G$, a contradiction. Thus $e$ is in $E\left(\operatorname{cl}\left(G^{1}\right)\right)$. Case (ii): Assume $y_{1} \in e$. Then there is $f \in E(\operatorname{cl}(G))$, with $x_{1} \in f$, such that $e=\left(f \backslash\left\{x_{1}\right\}\right) \cup\left\{y_{1}\right\}$. Since $\left\{x, x_{1}\right\} \in E(G)$ for any $x$ in $f \backslash\left\{x_{1}\right\}$, one has that $\left\{x, y_{1}\right\} \in E\left(G^{1}\right)$ for 
any $x$ in $f \backslash\left\{x_{1}\right\}$. Then $e$ is a clique of $G^{1}$. If $e$ is not a maximal clique of $G^{1}$, there is $x \notin e$ which is adjacent in $G$ to any vertex of $f \backslash\left\{x_{1}\right\}$ and $x$ is adjacent to $y_{1}$ in $G^{1}$. In particular $x \neq x_{1}$. Then $x$ is adjacent in $G$ to $x_{1}$ and consequently $x$ is adjacent in $G$ to any vertex of $f$, a contradiction because $f$ is a maximal clique of $G$. Thus $e$ is in $\operatorname{cl}\left(G^{1}\right)$. Next we prove the inclusion $E\left(\operatorname{cl}\left(G^{1}\right)\right) \subset E\left(\mathscr{C}^{1}\right)$. Take $e \in E\left(\operatorname{cl}\left(G^{1}\right)\right)$, i.e., $e$ is a maximal clique of $G^{1}$. Case (i): Assume $y_{1} \notin e$. Then $e$ is a maximal clique of $G$, and so an edge of $\mathscr{C}^{1}$. Case (ii): Assume $y_{1} \in e$. Then $e \backslash\left\{y_{1}\right\}$ is a clique of $G$ and $\left\{x, y_{1}\right\} \in E\left(G^{1}\right)$ for any $x$ in $e \backslash\left\{y_{1}\right\}$. Then $\left\{x, x_{1}\right\}$ is in $E(G)$ for any $x$ in $e \backslash\left\{y_{1}\right\}$. Hence $f=\left(e \backslash\left\{y_{1}\right\}\right) \cup\left\{x_{1}\right\}$ is a clique of $G$. Note that $f$ is a maximal clique of $G$. Indeed if $f$ is not a maximal clique of $G$, there is $x \in V(G) \backslash f$ which is adjacent in $G$ to every vertex of $e \backslash\left\{y_{1}\right\}$ and to $x_{1}$. Thus $x$ is adjacent to $y_{1}$ in $G^{1}$ and to every vertex in $e \backslash\left\{y_{1}\right\}$, i.e., $e \cup\{x\}$ is a clique of $G^{1}$, a contradiction. Thus $f \in \operatorname{cl}(G)$. Since $e=\left(f \backslash\left\{x_{1}\right\}\right) \cup\left\{y_{1}\right\}$ we obtain that $e \in E\left(\mathscr{C}^{1}\right)$.

Unfortunately we do not have an analogous version of Lemma 3.4 valid for a deletion. In other words, if $G$ is a graph, the equality $\operatorname{cl}(G)^{w}=\operatorname{cl}\left(G^{w}\right)$, with $w$ an integer vector, fails in general (see Remark 3.5).

Remark 3.5. Let $G$ be a graph. Let $G^{1}=G \backslash\left\{x_{1}\right\}$ (resp. $\operatorname{cl}(G)^{1}=$ $\left.\operatorname{cl}(G) \backslash\left\{x_{1}\right\}\right)$ be the graph (resp. clutter) obtained from $G$ (resp. $\left.\operatorname{cl}(G)\right)$ by deleting the vertex $x_{1}$. The equality $\operatorname{cl}(G)^{1}=\operatorname{cl}\left(G^{1}\right)$ fails in general. For instance if $G$ is a cycle of length three, then $E\left(\operatorname{cl}(G)^{1}\right)=\emptyset$ and $\operatorname{cl}\left(G^{1}\right)$ has exactly one edge.

Let $\mathscr{D}$ be a digraph, that is, $\mathscr{D}$ consists of a finite set $V(\mathscr{D})$ of vertices and a set $E(\mathscr{D})$ of ordered pairs of distinct vertices called edges. Let $A, B$ be two sets of vertices of $\mathscr{D}$. For use below recall that a (directed) path of $\mathscr{D}$ is called an $A-B$ path if it runs from a vertex in $A$ to a vertex in $B$. A set $C$ of vertices is called an $A-B$ disconnecting set if $C$ intersects each $A-B$ path. For convenience we recall the following classical result.

Theorem 3.6. (Menger's theorem, see [12, Theorem 9.1]) Let $\mathscr{D}$ be a digraph and let $A, B$ be two subsets of $V(\mathscr{D})$. Then the maximum number of vertex-disjoint $A-B$ paths is equal to the minimum size of an $A-B$ disconnecting vertex set.

We come to the main result of this section.

TheOREM 3.7. Let $P=(X, \prec)$ be a poset on the vertex set $X=\left\{x_{1}, \ldots, x_{n}\right\}$ and let $G$ be its comparability graph. If $\mathscr{C}=\operatorname{cl}(G)$ is the clutter of maximal cliques of $G$, then $\mathscr{C}$ satisfies the max-flow min-cut property.

Proof. We can regard $P$ as a transitive digraph without cycles of length 
two with vertex set $X$ and edge set $E(P)$, i.e., the edges of $P$ are ordered pairs $(a, b)$ of distinct vertices with $a \prec b$ such that:

(i) $(a, b) \in E(P)$ and $(b, c) \in E(P) \Rightarrow(a, c) \in E(P)$ and

(ii) $(a, b) \in E(P) \Rightarrow(b, a) \notin E(P)$.

Note that because of these two conditions, $P$ is in fact an acyclic digraph, that is, it has no directed cycles. Let $x_{1}$ be a vertex of $P$ and let $y_{1}$ be a new vertex. Consider the digraph $P^{1}$ with vertex set $X^{1}=X \cup\left\{y_{1}\right\}$ and edge set

$$
E\left(P^{1}\right)=E(P) \cup\left\{\left(y_{1}, x\right) \mid\left(x_{1}, x\right) \in E(P)\right\} \cup\left\{\left(x, y_{1}\right) \mid\left(x, x_{1}\right) \in E(P)\right\} .
$$

The digraph $P^{1}$ is transitive. Indeed let $(a, b)$ and $(b, c)$ be two edges of $P^{1}$. If $y_{1} \notin\{a, b, c\}$, then $(a, c) \in E(P) \subset E\left(P^{1}\right)$ because $P$ is transitive. If $y_{1}=a$, then $\left(x_{1}, b\right)$ and $(b, c)$ are in $E(P)$. Hence $\left(x_{1}, c\right) \in E(P)$ and $\left(y_{1}, c\right) \in E\left(P^{1}\right)$. The cases $y_{1}=b$ and $y_{1}=c$ are treated similarly. Thus $P^{1}$ defines a poset $\left(X^{1}, \prec^{1}\right)$. The comparability graph $H$ of $P^{1}$ is precisely the graph $G^{1}$ obtained from $G$ by duplicating the vertex $x_{1}$ by the vertex $y_{1}$. To see this note that $\{x, y\}$ is an edge of $G^{1}$ if and only if $\{x, y\}$ is an edge of $G$ or $y=y_{1}$ and $\left\{x, x_{1}\right\}$ is an edge of $G$. Thus $\{x, y\}$ is an edge of $G^{1}$ if and only if $x$ is related to $y$ in $P$ or $y=y_{1}$ and $x$ is related to $y$ in $P^{1}$, i.e., $\{x, y\}$ is an edge of $G^{1}$ if and only if $\{x, y\}$ is an edge of $H$. From Lemma 3.4 we get that $\operatorname{cl}(G)^{1}=\operatorname{cl}\left(G^{1}\right)$, where $\operatorname{cl}(G)^{1}$ is the clutter obtained from $\operatorname{cl}(G)$ by duplicating the vertex $x_{1}$ by the vertex $y_{1}$. Altogether we obtain that the clutter $\operatorname{cl}(G)^{1}$ is the clique clutter of the comparability graph $G^{1}$ of the poset $P^{1}$.

By Theorem 3.3 it suffices to prove that $\operatorname{cl}(G)^{w}$ has the König property for all $w \in \mathbf{N}^{n}$. Since duplications commute with deletions, by permuting vertices, we may assume that $w=\left(w_{1}, \ldots, w_{r}, 0, \ldots, 0\right)$, where $w_{i} \geq 1$ for $i=1, \ldots, r$. Consider the clutter $\mathscr{C}_{1}$ obtained from $\operatorname{cl}(G)$ by duplicating $w_{i}-1$ times the vertex $x_{i}$ for $i=1, \ldots, r$. We denote the vertex set of $\mathscr{C}_{1}$ by $X_{1}$. By successively applying the fact that $\operatorname{cl}(G)^{1}=\operatorname{cl}\left(G^{1}\right)$, we conclude that there is a poset $P_{1}$ with comparability graph $G_{1}$ and vertex set $X_{1}$ such that $\mathscr{C}_{1}=\operatorname{cl}\left(G_{1}\right)$. As before we regard $P_{1}$ as a transitive acyclic digraph.

Let $A$ and $B$ be the set of minimal and maximal elements of the poset $P_{1}$, i.e., the elements of $A$ and $B$ are the sources and sinks of $P_{1}$ respectively. We set $S=\left\{x_{r+1}, \ldots, x_{n}\right\}$. Consider the digraph $\mathscr{D}$ whose vertex set is $V(\mathscr{D})=X_{1} \backslash S$ and whose edge set is defined as follows. A pair $(x, y)$ in $V(\mathscr{D}) \times V(\mathscr{D})$ is in $E(\mathscr{D})$ if and only if $(x, y) \in E\left(P_{1}\right)$ and there is no vertex $z$ in $X_{1}$ with $x \prec z \prec y$. Notice that $\mathscr{D}$ is a sub-digraph of $P_{1}$ which is not necessarily the digraph of a poset. We set $A_{1}=A \backslash S$ and $B_{1}=B \backslash S$. Note that $\mathscr{C}^{w}=\mathscr{C}_{1} \backslash S$, the clutter obtained from $\mathscr{C}_{1}$ by removing all vertices of $S$ and all edges sharing a vertex with $S$. If every edge of $\mathscr{C}_{1}$ intersects $S$, then $E\left(\mathscr{C}^{w}\right)=\emptyset$ and there is nothing to prove. Thus we may assume that there is a maximal clique $K$ of $G_{1}$ 
disjoint form $S$. Note that by the maximality of $K$ and by the transitivity of $P_{1}$ we get that $K$ contains at least one source and one sink of $P_{1}$, i.e., $A_{1} \neq \emptyset$ and $B_{1} \neq \emptyset$ (see argument below).

The maximal cliques of $G_{1}$ not containing any vertex of $S$ correspond exactly to the $A_{1}-B_{1}$ paths of $\mathscr{D}$. Indeed let $c=\left\{v_{1}, \ldots, v_{s}\right\}$ be a maximal clique of $G_{1}$ disjoint from $S$. Consider the sub-poset $P_{c}$ of $P_{1}$ induced by $c$. Note that $P_{c}$ is a tournament, i.e., $P_{c}$ is an oriented graph (no-cycles of length two) such that any two vertices of $P_{c}$ are comparable. By [1, Theorem 1.4.5] any tournament has a Hamiltonian path, i.e., a spanning oriented path. Therefore we may assume that

$$
v_{1} \prec v_{2} \prec \cdots \prec v_{s-1} \prec v_{s}
$$

By the maximality of $c$ we get that $v_{1}$ is a source of $P_{1}, v_{s}$ is a sink of $P_{1}$, and $\left(v_{i}, v_{i+1}\right)$ is an edge of $\mathscr{D}$ for $i=1, \ldots, s-1$. Thus $c$ is an $A_{1}-B_{1}$ path of $\mathscr{D}$, as required. Conversely let $c=\left\{v_{1}, \ldots, v_{s}\right\}$ be an $A_{1}-B_{1}$ path of $\mathscr{D}$. Clearly $c$ is a clique of $P_{1}$ because $P_{1}$ is a poset. Assume that $c$ is not a maximal clique of $G_{1}$. Then there is a vertex $v \in X_{1} \backslash c$ such that $v$ is related to every vertex of $c$. Since $v_{1}, v_{s}$ are a source and a sink of $P_{1}$ respectively we get $v_{1} \prec v \prec v_{s}$. We claim that $v_{i} \prec v$ for $i=1, \ldots, s$. By induction assume that $v_{i} \prec v$ for some $1 \leq i<s$. If $v \prec v_{i+1}$, then $v_{i} \prec v \prec v_{i+1}$, a contradiction to the fact that $\left(v_{i}, v_{i+1}\right)$ is an edge of $\mathscr{D}$. Thus $v_{i+1} \prec v$. Making $i=s$ we get that $v_{s} \prec v$, a contradiction. This proves that $c$ is a maximal clique of $G_{1}$. Therefore, since the maximal cliques of $G_{1}$ not containing any vertex in $S$ are exactly the edges of $\mathscr{C}^{w}=\mathscr{C}_{1} \backslash S$, by Menger's theorem (see Theorem 3.6) we obtain that $\beta_{1}\left(\mathscr{C}^{w}\right)=\alpha_{0}\left(\mathscr{C}^{w}\right)$, i.e., $\mathscr{C}^{w}$ satisfies the König property.

Let $G$ be a graph. The matrix $A$ whose column vectors are the characteristic vectors of the maximal cliques of $G$ is called the vertex-clique matrix of $G$. It is well known that if $G$ is a comparability graph and $A$ is the vertex-clique matrix of $G$, then $G$ is perfect [12, Corollary 66.2a] and the polytope

$$
P(A)=\{x \mid x \geq 0 ; x A \leq \mathbf{1}\}
$$

is integral [12, Corollary 65.2e]. The next result complement this fact.

Corollary 3.8. Let $G$ be a comparability graph and let $A$ be the vertexclique matrix of $G$. Then the polyhedron $Q(A)=\{x \mid x \geq 0 ; x A \geq \mathbf{1}\}$ is integral.

Proof. By Theorem 3.7 the clique $\operatorname{clutter} \operatorname{cl}(G)$ has the max-flow mincut property. Thus the system $x A \geq \mathbf{1} ; x \geq 0$ is totally dual integral, i.e., the maximum in Eq. (3) has an integer optimum solution $y$ for each integer 
vector $w$ with finite maximum. Hence $Q(A)$ has only integer vertices by [12, Theorem 5.22].

\section{Normally torsion freeness and normality}

Let $R=K\left[x_{1}, \ldots, x_{n}\right]$ be a polynomial ring over a field $K$, let $\mathscr{C}$ be a clutter on the vertex set $X=\left\{x_{1}, \ldots, x_{n}\right\}$, and let $I=I(\mathscr{C}) \subset R$ be the edge ideal of $\mathscr{C}$. Recall that $\mathfrak{p}$ is a minimal prime of $I$ if and only if $\mathfrak{p}=(C)$ for some minimal vertex cover $C$ of $\mathscr{C}$ [15, Proposition 6.1.16]. Thus if $C_{1}, \ldots, C_{s}$ are the minimal vertex covers of $\mathscr{C}$, then the primary decomposition of $I$ is

$$
I=\mathfrak{p}_{1} \cap \mathfrak{p}_{2} \cap \cdots \cap \mathfrak{p}_{s},
$$

where $\mathfrak{p}_{i}$ is the prime ideal of $R$ generated by $C_{i}$. The $i$ th symbolic power of $I$, denoted by $I^{(i)}$, is given by $I^{(i)}=\mathfrak{p}_{1}^{i} \cap \cdots \cap \mathfrak{p}_{s}^{i}$.

TheOREm 4.1 ([7]). Let $\mathscr{C}$ be a clutter, let $A$ be the incidence matrix of $\mathscr{C}$, and let $I=I(\mathscr{C})$ be its edge ideal. Then the following are equivalent:

(i) I is normal and $Q(A)=\{x \mid x \geq 0 ; x A \geq \mathbf{1}\}$ is an integral polyhedron.

(ii) $I$ is normally torsion free, i.e., $I^{i}=I^{(i)}$ for $i \geq 1$.

(iii) $\mathscr{C}$ has the max-flow min-cut property.

There are some other nice characterizations of the normally torsion free property that can be found in [6], [9].

Our main algebraic result is:

THEOREM 4.2. If $G$ is a comparability graph and $\mathrm{cl}(G)$ is its clique clutter, then the edge ideal $I=I(\mathrm{cl}(G))$ of $\mathrm{cl}(G)$ is normally torsion free and normal.

Proof. It follows from Theorems 3.7 and 4.1.

\section{Complete admissible uniform clutters}

In this paragraph we introduce a family of clique clutters of comparability graphs. Let $d \geq 2, g \geq 2$ be two integers and let

$$
X^{1}=\left\{x_{1}^{1}, \ldots, x_{g}^{1}\right\}, X^{2}=\left\{x_{1}^{2}, \ldots, x_{g}^{2}\right\}, \ldots, X^{d}=\left\{x_{1}^{d}, \ldots, x_{g}^{d}\right\}
$$

be disjoint sets of variables. The clutter $\mathscr{C}$ with vertex set $X=X^{1} \cup \cdots \cup X^{d}$ and edge set

$$
E(\mathscr{C})=\left\{\left\{x_{i_{1}}^{1}, x_{i_{2}}^{2}, \ldots, x_{i_{d}}^{d}\right\} \mid 1 \leq i_{1} \leq i_{2} \leq \cdots \leq i_{d} \leq g\right\}
$$

is called a complete admissible uniform clutter. The edge ideal of this clutter was introduced and studied in [5]. This ideal has many good properties, for 
instance $I(\mathscr{C})$ and its Alexander dual are Cohen-Macaulay and have linear resolutions (see [5, Proposition 4.5, Lemma 4.6]). For a thorough study of Cohen-Macaulay admissible clutters see [8], [10].

THEOREM 4.3. If $\mathscr{C}$ is a complete admissible uniform clutter, then its edge ideal $I(\mathscr{C})$ is normally torsion free and normal.

Proof. Let $P=(X, \prec)$ be the poset with vertex set $X$ and partial order given by $x_{k}^{\ell} \prec x_{p}^{m}$ if and only if $1 \leq \ell<m \leq d$ and $1 \leq k \leq p \leq g$. We denote the comparability graph of $P$ by $G$. We claim that $E(\mathscr{C})=E(\operatorname{cl}(G))$, where $\operatorname{cl}(G)$ is the clique clutter of $G$. Let $f=\left\{x_{i_{1}}^{1}, x_{i_{2}}^{2}, \ldots, x_{i_{d}}^{d}\right\}$ be an edge of $\mathscr{C}$, i.e., we have $1 \leq i_{1} \leq i_{2} \leq \cdots \leq i_{d} \leq g$. Clearly $f$ is a clique of $G$. If $f$ is not maximal, then there is a vertex $x_{k}^{\ell}$ not in $f$ which is adjacent in $G$ to every vertex of $f$. In particular $x_{k}^{\ell}$ must be comparable to $x_{i_{\ell}}^{\ell}$, which is impossible. Thus $f$ is an edge of $\operatorname{cl}(G)$. Conversely let $f$ be an edge of $\operatorname{cl}(G)$. We can write $f=\left\{x_{i_{1}}^{k_{1}}, x_{i_{2}}^{k_{2}}, \ldots, x_{i_{s}}^{k_{s}}\right\}$, where $k_{1}<\cdots<k_{s}$ and $i_{1} \leq \cdots \leq i_{s}$. By the maximality of $f$ we get that $s=d$ and $k_{i}=i$ for $i=1, \ldots, d$. Thus $f$ is an edge of $\mathscr{C}$. Hence by Theorem 4.2 we obtain that $I(\mathscr{C})$ is normally torsion free and normal.

ACKNOWLEDGMENTS. We thank Seth Sullivant for pointing out an alternative proof of Theorem 4.2 based on the fact that the edge ideal of a comparability graph is differentially perfect (see [13, Section 4]). One of the consequences of being differentially perfect is that if every maximal chain in the poset has the same fixed length $k$, then the edge ideal of the clique clutter of its comparability graph is normally torsion free. The general case, that is, the situation where not all maximal chains have the same length, can be reduced to the special case above. We also thank Alexander Schrijver for pointing out [12, Theorem 14.18] and the characterization of the max-flow min-cut property given in Theorem 3.3. Finally, we thank the referee for a careful reading of the paper and for pointing out [5] as an earlier reference for complete admissible uniform clutters. The authors have been informed that Corollary 2.5 was observed by Ngô Viêt Trung when $I$ is the edge ideal of a hypergraph.

\section{REFERENCES}

1. Bang-Jensen, J., and Gutin, G., Digraphs. Theory, Algorithms and Applications, 2nd printing, Springer Monographs in Math., Springer, London 2006.

2. Baum, S., and Trotter, L. E., Integer rounding for polymatroid and branching optimization problems, SIAM J. Algebraic Discrete Methods 2 (1981), 416-425.

3. Bruns, W., and Herzog, J., Cohen-Macaulay Rings, revised edition, Cambridge Studies in Advanced Math. 39, Cambridge Univ. Press, Cambridge 1997. 
4. Escobar, C. A., Villarreal, R. H., and Yoshino, Y., Torsion freeness and normality of blowup rings of monomial ideals, pp. 69-84 in: Commutative Algebra, Lect. Notes Pure Appl. Math. 244, Chapman \& Hall/CRC, Boca Raton, FL 2006.

5. Fløystad, G., and Vatne, J.E.,(Bi-)Cohen-Macaulay simplicial complexes and their associated coherent sheaves, Comm. Algebra 33 (2005), 3121-3136.

6. Gitler, I., Reyes, E., and Villarreal, R. H., Blowup algebras of square-free monomial ideals and some links to combinatorial optimization problems, Rocky Mountain J. Math. 39 (2009), 71-102.

7. Gitler, I., Valencia, C. E., and Villarreal, R. H., A note on Rees algebras and the MFMC property, Beiträge Algebra Geom. 48 (2007), 141-150.

8. Hà, H. T., Morey, S., and Villarreal, R. H., Cohen-Macaulay admissible clutters, J. Commut. Algebra, to appear.

9. Huneke, C., Simis, A., and Vasconcelos, W. V., Reduced normal cones are domains, pp. 95101 in: Invariant Theory, Proc. Denton 1986, Contemp. Math. 88, Amer. Math. Soc., Providence, RI 1989.

10. Morey, S., Reyes, E., and Villarreal, R. H., Cohen-Macaulay, shellable and unmixed clutters with a perfect matching of König type, J. Pure Appl. Algebra 212 (2008), 1770-1786.

11. Schrijver, A., Theory of Linear and Integer Programming, Wiley, New York 1986.

12. Schrijver, A., Combinatorial Optimization, Algorithms and Combinatorics 24, Springer, Berlin 2003.

13. Sullivant, S., Combinatorial symbolic powers, J. Algebra 319 (2008), 115-142.

14. Vasconcelos, W. V., Integral Closure, Springer Monographs in Math., Springer, New York 2005.

15. Villarreal, R. H., Monomial Algebras, Monographs and Textbooks in Pure and App. Math. 238, Marcel Dekker, New York 2001.

DEPARTAMENTO DE MATEMÁTICAS

CENTRO DE INVESTIGACIÓN Y DE ESTUDIOS AVANZADOS DEL IPN

APARTADO POSTAL 14-740

07000 MÉXICO CITY, D.F.

MEXICO

E-mail: 1dupont@math.cinvestav.mx, vila@math.cinvestav.mx 